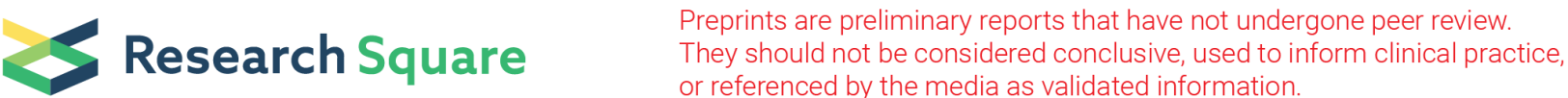

\section{Sevoflurane Exposure of Clinical Dose in Pregnant Rat Induces Vcan Changes Without Significant Neural Apoptosis in the Offspring}

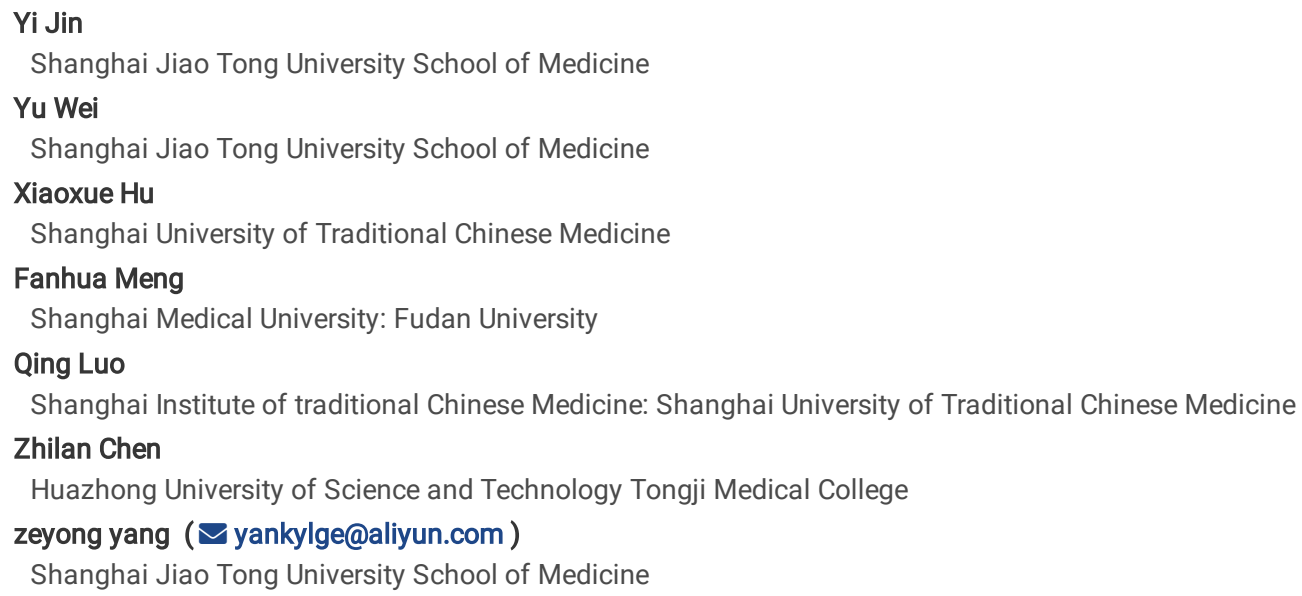




\section{Abstract}

Background: Sevoflurane is a commonly used inhalational anesthetic in clinic. Prolonged exposure to sevoflurane can induce significant changes in lipid metabolism and neuronal damage in the developing brain. However, the effect of sevoflurane exposure of clinical dose in pregnant rat remains unclear.

Results: The following study was to explore lipidomics and transcriptomics changes related to $2 \%$ sevoflurane exposure for $6 \mathrm{~h}$ in the developing brain of newborn offspring rats. UPLC/TOF-MS(Ultra Performance Liquid Chromatography/Time-Of-Flight Mass Spectrometry) and RNA-seq analysis was used to acquire metabolomics and transcriptomics profiles. We used RNA sequencing (RNA-seq) to analyze the expression of the coding and non-coding transcripts in neural cells of cerebral cortex. No significant differences in arterial oxygen tension $\left(\mathrm{PaO}_{2}\right)$, arterial carbon dioxide tension $(\mathrm{PaCO})$, arterial blood gas were found between groups. RSDs(Relative Standard Deviation) of retention times were $<1.53 \%$, and RSDs of peak areas ranged from $2.13 \%$ to $8.51 \%$. BPC profiles showed no differences between groups. We evaluated the Partial Least Square-Discriminant Analysis (PLS-DA) model: in negative ion mode: R2X, over 70\%: R2Y, over 93\%: Q2 (cum), over 80\%; Cell apoptosis was not remarkably enhanced by TUNEL and HE staining in sevoflurane exposure in comparison to control group $(P>0.05)$. Glycerophospholipid and sphingolipid metabolism disturbance might have adverse influence on neurodevelopment in offspring. The expression of mRNAs (Vcan gene, related to neuronal development, function, and repair) of sevoflurane group were significantly increased in the differential genes by qRT-PCR verification.

Conclusions: glycerophospholipid and sphingolipid metabolism homeostasis may be potential therapeutic approaches against inhalational anestheticsinduced neurodegenerative disorders. Meanwhile, sevoflurane induced Vcan changes, which indicate some lipidomics and transcriptomics changes was happened even if neural cell apoptosis was not significantly changed in usual clinical dose of sevoflurane exposure.

\section{Introduction}

Each year, over 10 million pregnant women in China implement anesthesia. Maternal sevoflurane exposure may present a possible risk for neurodevelopment in her offspring [1]. Furthermore, preclinical studies have indicated that a prolonged exposure to inhalational anesthetics during brain synaptogenesis can hurt the immature central nervous system leading to standing neurocognitive impairments [1]. Prolonged sevoflurane inhalation has shown to decrease phosphatidylethanolamine, phosphatidylserine, and phosphatidylglycerol, and to increase 4-hydroxynonenal (relevant to stress and lipid); phosphatidylserine is involved in signaling pathways relevant to neural cell synaptogenesis, survival and neurite growth[2-6]. Alpha-lipoic acid suppresses sevofluraneassociated neural cell apoptosis by PI3K/Akt pathway [7].

In addition, sevoflurane with high concentration and long-term exposure have shown to alter metabolism of glucose, amino acid, and lipid, as well as intracellular antioxidant and osmotic substance in developmental nerve [8]. Lipid changes may disturb phospholipid homeostasis, change the integrity, orientation, permeability and function of the membrane, leading to neurological dysfunction and degeneration. Meanwhile, RNA-Seq can detect alterations of expression of low abundance transcripts. Can sevoflurane exposure in pregnant rat induce potential neurotoxicity in the offspring including lipidomics and RNA-Seq changes?

We hypothesized that sevoflurane exposure can induce lipidomics and transcriptomics changes of neurotoxicity in the developing brain in offspring rats after birth. To the best of our knowledge, this is the first study to explore the lipid and transcriptomics changes of anesthetics-induced neurotoxicity in rats. As we have known, it is the first study of neurotoxic lipid and transcriptional changes induced by anesthetics.

\section{Methods}

\section{Animals}

All animal studies, containing rats euthanasia procedures, were approved and complied with AALAC and IACUC guidelines and guidelines for institutional animal care in Shanghai Jiao Tong University (Permit number: A2016077). Sixteen SD rats weighing 400-500 g within 18 days of gestation were bought from IACUC of Shanghai Jiao Tong University.

All rats did not carry viruses, bacteria and parasites. Animal health were continuously recorded by a sentinel program. Rats were kept in Individual ventilated plastic cages $(420 \mathrm{~mm} \times 250 \mathrm{~mm} \times 230 \mathrm{~mm}$ ). A standard cardbox house was provided, autoclaved hay (about $8 \otimes 12 \mathrm{~g} / \mathrm{cage}$ ) and two Nestlets $^{\text {TM }}$ (about $5 \times 5 \mathrm{~cm}$ ) were supplied. Rats were fed a pelleted rat diet by Jiangsu cooperative medical bio-engineering co..LTD and ordinary pure water after high temperature sterilization. The rats were housed in an environment with temperature of $23 \pm 2^{\circ} \mathrm{C}$, relative humidity of $50 \pm 15 \%$ and a light/dark cycle of $12 / 12 \mathrm{hr}$ (approximately $20 \mathrm{~lx}$ in the cage), the air pressure was controlled at $10 \mathrm{~Pa}$. HPLC(High Performance Liquid Chromatography)-grade acetonitrile and formic acid were purchased from Merck (USA), calibration solution for TOF-MS was bought from AB SCIEX (AB SCIEX,Foster City, USA), ultra-pure water was generated by a Milli-Q pure water system (Millipore,USA).

\section{Experimental Protocol}

Sprague Dawley (SD) pregnant rats, 3 months old, 400-500g weighing, were randomly divided in: sevoflurane group (S) or a control group (C) at gestational day $(\mathrm{G})$ 18. Eight pregnant rats in each group were kept in a temperature humidity -controlled room with a 12-hour period of light and darkness from 3:00 to 15:00, The anesthesia chamber was given $2 \%$ sevoflurane and $100 \%$ oxygen for 6 hours in sevoflurane group. Control group was given $100 \%$ oxygen at the same flow rate in the same chamber for 6 hours. Parameter setting is $400 \mathrm{ml} / \mathrm{min}$ as the total gas flow. A gas analyzer (Drager, Lubeck, Germany) sustained to monitor oxygen, carbon dioxide and sevoflurane concentration. The rats kept with spontaneous breath. After sevoflurane termination, the rats were placed in a chamber containing 100\% oxygen for 20 min. Pregnant mice arterial blood sample were collected and analyzed by i-STAT 1 Analyzer (MN: 300-G, Abbott Park, USA) immediately after termination of anesthesia. The control group (C) received 100\% oxygen at an identical flow rate for $6 \mathrm{~h}$ in an identical chamber. 
Remaining rats were kept in the chamber for delivery, the offspring of sevoflurane group and control group were 40 and 42 , respectively. A total of 24 (7postnatal) rats were randomly selected from offspring rats of two groups ( $n=12$ in each). Serum samples of offspring rats were frozen at $-80^{\circ} \mathrm{C}$ for next analysis. In our study, the relevant details was as follows regarding the euthanization of study animals. Pregnant rats were sacrificed by cutting off the abdominal aorta under sevoflurane anesthesia or increasing the concentration of carbon dioxide in accordance with the animal laboratory's euthanasia method when they were taken blood from the abdominal aorta directly, The newborn rats were placed in a anesthetic box with a concentration of about $3 \%$ and then did decollation under anesthesia, Other rats of the experiments implemented euthanasia by increasing the concentration of carbon dioxide in accordance with the animal laboratory's euthanasia method.

\section{Sample preparation and Ultra Performance Liquid Chromatography (UPLC) Analysis}

Sample pretreatment requirement: $100 \mu \mathrm{l}$ of neonatal rat serum (from sevoflurane and control group) was placed in a tube (pretreated with heparinization). Adding paclitaxel storage solution $(100 \mu \mathrm{g} / \mathrm{ml}) 15 \mu \mathrm{l}$ as internal reference material, then got methanol $300 \mu \mathrm{l}$, vortex oscillation 3 min, high-speed centrifugation $(12000 \mathrm{r} / \mathrm{min})$ centrifugation $\left(4^{\circ} \mathrm{C}, 10 \mathrm{~min}\right)$, The supernatant of precision absorption was $200 \mu \mathrm{l}$, and the microporous filtration membrane $(0.22 \mu \mathrm{m})$ was to be used. Chromatographic conditions: Waters Xterra MS C 8 column $(2.1 \times 100 \mathrm{~mm}, 3.5 \mu \mathrm{m})$. The mobile phase A: acetonitrile: isopropanol $(5: 2, \mathrm{v} / \mathrm{v})$ contains $2 \mathrm{mmol} / \mathrm{L}$ ammonium acetate, $0.1 \%$ formic acid and $0.1 \%$ formic acid aqueous solution containing $2 \mathrm{mmol} / \mathrm{L}$ ammonium acetate. Gradient elution procedure: 0-1 $\min 10 \%$ A, 1 / 2 min 10-30\% A, 2 / $4 \min 30$ / 50\% A, 4 / 8 min 50 / 70\% A, 8 / 12 min 70 / 100\%, 1 / 2-24 min 100\% A, 24-24.5 min 100$10 \%$ A, 24.5-30 min 10\% A. Flow rate: $0.35 \mathrm{~mL} / \mathrm{min}$. Column temperature: $40{ }^{\circ} \mathrm{C}$. Sample volume: $10 \mu \mathrm{L}$. The method was used to analyze other lipid compounds (glyceryl ester, glycerol phospholipid, high abundance sphingolipid). The related substances were identified by ultra-high performance liquid chromatography (HPLC) with multi-reaction monitor.

\section{Pattern recognition analysis based on PLS-DA}

The score map was obtained by pattern recognition, and then the model was evaluated, the candidate heteron ions were added to the peptide segment. The (VIP) value of the variable projection importance marker in the PLS-DA model was used to screen the candidate differential protein. The partial least squares discriminant analysis (PLS-DA) was used to excavate the changes among different samples to determine the key ingredients. After dealing with the data, the data matrix containing sample variables was obtained, and the next metabolite was further analyzed. Compounds with projected value $p<0.05$ and $>1.0$ could be identified as potential biomarkers from the PLS-DA model.

\section{Hematoxylin and Eosin (HE) Staining}

HE staining was use to detect the neural cell apoptosis in both hippocampus and cerebral cortex of neonatal rats. HE staining: sections were separated, then treated by hydration, hematoxylin staining, $1 \%$ ethanol hydrochloric acid alcohol differentiation for $1 \mathrm{~s}$ and eosin staining. The stained slices were dehydrated, encased in neutral balm, and covered with a coverslip. The microscope (Olympus) got the image at $400 \mathrm{x}$ magnification.

\section{TUNEL staining}

Sections of the hippocampus and cerebral cortex of each group were frozen and soaked in $3 \% \mathrm{H} 2 \mathrm{O} 2$ phosphate buffer (PBS) to eliminate the endogenous peroxidase reaction. Washed 3 times, and 5 min once, then $20 \%$ fetal bovine serum (FBS) and 3\% FBS protein were put into it for 15 min. After the TUNEL reaction solution was connected with fluorescein, the slices were placed in a humidifying box at $37{ }^{\circ} \mathrm{C}$ for 1 hour, then washed with PBS three times for 5 minutes. Then, the parts are re-evaluated. The termination solution at room temperature acted for 10 minutes, and the anti-digoxin peroxidase antibody acted for 30 minutes at $37^{\circ} \mathrm{C}$. It was washed three times with PBS for 5 minutes. Afterwards, The slices were made by 3,3'-diaminobenzidine, dyed by HE, transparent by xylene and sealed by neutral resin. The sections were then put down a fluorescence microscope. The TUNEL positive staining was observed under the S BX 51 Upright fluorescence microscope ( 400-fold, USA). The experiment was repeated four times. The apoptotic rate was calculated. The formula was that: apoptotic rate $=$ TUNEL positive cells $/$ the total cells.

\section{RNA-seq and Real-time PCR (RT-PCR) Verification}

The RNA was extracted by Trizol methods from the cortical cells $\left(10^{2}\right.$ to $\left.10^{4}\right)$ of the neonatal rats include sevoflurane group and control group, and we added $800 \mu$ I TRIZOL reagent to the sample. After the sample was lysed, the samples were separated by adding chloroform. Before adding isopropanol to precipitate RNA, 5-10 $\mu \mathrm{g}$ RNA-free glycogen was added as aqueous phase carrier. In order to reduce the viscosity of the solution, the sample was cut through the 26 needles twice before chloroform was added to cut the genomic DNA. After two-phase separation, glycogen was left in the aqueous phase and co-precipitated with RNA. The real-time quantitative PCR reaction system consisted of $1 \mu \mathrm{l} c D N A, 1 \mu l$ upstream and downstream primer mixture, $5 \mu l$ premixed solution and $3 \mu \mathrm{l}$ actinase water. Follow-up database construction and sequence analysis of full transcript was set by Cloudseq Biotech (Shanghai) Co.,Ltd. Relevant test for quality and RNA-seq sequencing was finished by above-mentioned company. The target gene RT-PCR was detected in SYBR Green (TaKaRa) system (LightCycler 480) when it was needed. Instruments included primer design software: Primer 5.0. and ViiA 7 Real-time PCR System (Applied Biosystems). The expression of related genes was administrated by quantitative RT-PCR for the verification of the significant gene, which was performed by the relevant instructions. To verify the accuracy based on the analysis of RNA-seq, the differential genes in relevant signaling pathway were tested in the samples. The gene relative expression was calculated, and GAPDH was used as internal reference for relative quantitative analysis.

\section{Statistical Analysis}

Data analyses were derived from using Graph Prism 5.0 software. Assumptions of normality and homogeneity of variance were first checked. Relevant data were expressed as the mean \pm standard deviation. The independent samples $t$-test were used to analyze the differences between groups for continuous measures including Tunel or HE staining data. Multigroup comparisons of the means were carried out with Tukey post hoc tests. And a Partial Least Squares 
Discriminant Analysis (PLSDA) in MatLab (version 3.7.1) was used. There were statistically significant differences at a standard of $P \otimes 0.05$, The target genes candidates of DE miRNAs were used to KEGG enrichment analysis and pathway database [31-33]. Kobas [34] software is used to evaluate the statistical enrichment of candidate genes of KEGG pathway. We used QValue [35] to adjust the P value. Qvalue $<0.01$ and | log2 (Foldchange) | $>1$ are set as the standard of remarkably differential expression (DE). Pathways were identified to be significantly enriched when FDR<0.05.

\section{Results}

Arterial blood gas ( $A B G$ ) test : Rats treated with sevoflurane in $S$ group and $C$ group performed $A B G$ test here to eliminate the possibility of blood gas alterations. As shown in Supplemental Table 1, $\mathrm{PH}$ value, partial pressure of oxygen $\left(\mathrm{PaO}_{2}\right)$ and partial pressure of carbon dioxide $\left(\mathrm{PaCO} \mathrm{C}_{2}\right)$ displayed no significant variance between two groups. Rats in S group were only affected by sevoflurane but not alterations in arterial blood gas caused by sevoflurane anesthesia.

\section{UPLC/TOF-MS analysis of sevoflurane-induced potential neurotoxicity to acquire metabolomics profiles}

UPLC/TOF-MS analysis was performed in both positive and negative ionization modes to acquire metabolomics profiles. Representative base peak chromatogram (BPC) was presented in Figure 1A and 1B. Method validation was conducted on six QC samples. Six common peaks in both negative and positive ion mode (paired retention time $\mathrm{M} / \mathrm{Z}$ ) variances of the retention time and peach area of ten bio-markers (Supplemental Table 2) were randomly selected and further examined for validation. Furthermore, RSDs of retention times were $<1.53 \%$ and RSDs of peak areas ranged from $2.13 \%$ to $8.51 \%$, which indicated that the proposed method of UPLC/TOF-MS profiling was acceptable. Variable importance in the projection (VIP) in PLS-DA is commonly used to discover the relevant biomarkers of neurotoxicity. Ten endogenous metabolites were identified as potential biomarkers in this study (Supplemental Table 2). Most biomarkers have been reported to be associated with some neurodegenerative diseases or neurodevelopment impairment (Supplemental Table 2, related pathways or diseases). Interestingly, these biomarkers were detected in the control (C) group , but not in the sevoflurane exposure (S) Group.

\section{Partial least squares-discriminate analysis (PLS-DA) and Lipidic metabolic pathway analysis of sevoflurane treatment}

Principal component analysis (PCA) was performed using UPLC/TOF-MS sample profiles in both groups, nevertheless, poor separation was obtained (data not shown). Consequently, partial least squares-discriminate analysis (PLS-DA), a supervised method, was then performed. A much better separation was acquired in positive ion mode (Figure $2 A$ ). Three parameters were used to evaluate the performance of PLS-DA model: in positive ion mode: $R^{2} X=0.665$, $R^{2} Y=0.996, Q^{2}=0.962$, so PLS-DA model were acceptable. The identified biomarkers were further analyzed with using MetaboAnalyst (http://www.metaboanalyst.ca). Briefly, glycerophospholipid metabolism was the most important metabolic pathway (Figure 2A). Furthermore, six metabolic pathways, including glycerophospholipid metabolism, glycosylphosphatidylinositol (GPI)-anchor biosynthesis, linoleic acid metabolism, alpha-Linolenic acid metabolism, sphingolipid metabolism and arachidonic acid metabolism were highlighted (Figure 2B).

\section{HE staining and Tunel staining}

Observation of TUNEL and HE staining for the morphological changes in each group $(\times 400)$ was administrated, neuronal apoptosis was detected by TUNEL and HE staining from hippocampi and cerebral cortex tissue of each offspring rat (Figure 3A, 3B). Cell apoptosis in the hippocampus and cerebral cortex tissue of sevoflurane-induced offspring rats was detected, however, the number of apoptotic cells in Tunel staining was not significantly increased in the sevoflurane group compared with the control and blank group $(P>0.05)$ (Figure 3C, 3D), which indicated cell apoptosis was happened in sevoflurane clinical dosage, but there was no significant difference in sevoflurane group in comparison with control group.

\section{Vcan gene RT-PCR changes and melting and amplification curves.}

Figure 4. Vcan gene RT-PCR changes(Figure 4A), included melting(Figure 4B), amplification(Figure 4C) and standard curves(Figure 4D). The Vcan gene provides instructions for making a protein called versican. Versican is a type of protein known as a proteoglycan, which means it has several sugar molecules attached to it. The overexpression have implications for understanding how Vcan regulates the toxicity of cerebral cortex including neural development, function, and repair.

\section{Significantly differentially expressed gene cluster thermogram}

We investigated differential expression gene cluster map(Figure 5A), Hierarchical clustering is a clustering method that is widely applied to gene expression data. Genes with similar expression patterns and samples with similar biological properties converge into a cluster. FPKM values of significantly expressed genes obtained by comparison between groups are used for cluster analysis, mRNA scatter diagram (Figure 5B) Pearson correlation 0.954. Up-regulated genes (640), not differential expression(10377), down-regulated genes(1810) for sevoflurane group and control group, Six hundred and forty genes were increased and 1810 genes were decreased in sevoflurane group compared with control group, mRNA Volcanic map(Figure 5C), Up-regulated genes (589), not differential expression(10531), down-regulated genes(1707) for sevoflurane group and control group, Red represents upregulation of significantly differentially expressed genes, while green represents down-regulation of significantly differentially expressed genes. RNA-seq experimental flow chart was shown in Figure 5D .

\section{Enrichment analysis of KEGG Pathway}

Figure 3.6. The first 20 KEGG pathways enriched by target genes of differentially expressed(DE) miRNA in 2 groups(Figure 6A), indicating that the RNA transport was shown a significant change by sevoflurane exposure. The terms of GO enrichment in Up signal pathway of DE gene exhibited significant differences for sevoflurane group and control group. Down signal pathway of DE gene was shown for sevoflurane group and control group(Figure 6B). Up Differentially Expressed Genes KEGG Pathway (Figure 6C) included IL-17 signaling pathway, Malaria, Osteoclast_differentiation, Pertussis, Salmonella 
infection, Spliceosome, Ribosome, RNA transport, Alzheimer's disease and NOD-like receptor signaling pathway. KEGG (Kyoto Encyclopedia of Genes and Genomes) results showed that relevant genes were differently expressed. KEGG pathway enrichment analysis was conducted to functionally categorize the target genes. All the putative target genes for DE miRNAs were enriched to the KEGG pathway. Every target gene of DE miRNAs was enriched into the KEGG pathway by functional classification of the target gene through KEGG pathway enrichment analysis. We used RNA-seq detection of transcriptomics technique to explore gene expression to supply with novel perspectives of the relevant signal pathway of sevoflurane exposure, which led to widespread and statistically significant gene changes to numerous transcripts involved in diverse pathways.

\section{Discussion}

Our data demonstrates that glycerophospholipid is an important biomarker, which comes from glycerophospholipid(http://www.genome.jp/dbget-bin) in glycerophospholipid metabolic process. Glycerophospholipids (GP) contain the main lipid category of mammalian cell membranes [9] which play an important role in cellular functions. The deregulation of lipids has shown to be associated with Alzheimer's, Parkinson's and Huntington diseases [10]. In our previous studies, we have demonstrated that sevoflurane induces significant damage in neural stem cells (FNSCs)[11]. Isoflurane induces cell apoptosis when used in high concentrations [12]. Sevoflurane exposure had harmful influences on neural development during embryonic phase [13-15]. Another study have also shown that neurotoxicity emerges from lysosphingolipids inhibiting PKC(Protein Kinase C). Membrane lipids, such as gangliosides and sphingolipids, are known to interact with both soluble $A \beta$ [16] and insoluble forms [17] and to affect $A \beta$ neural toxicity.

In our study, systems of MetaboAnalyst(http://www.metaboanalyst.ca) was used to analyzed further pathways of the identified biomarkers. Six metabolic pathways were highlighted, including glycerophospholipid metabolism, glycosylphosphatidylinositol (GPI)-anchor biosynthesis, linoleic acid metabolism, alpha-Linolenic acid metabolism, sphingolipid metabolism and arachidonic acid metabolism. A combination of these methods with stable isotope labeling is perfectly suitable for analyzing the metabolism of lipid species [18]. Meanwhile, we found some morphological changes in histopathological and immunohistochemical evaluation of hippocampi and cerebral cortex tissue of each offspring rat by HE and Tunel staining in sevoflurane group, however, the number of apoptotic cells was not significantly increased by TUNEL staining compared with the control group, which indicate that the neural cell apoptosis is not very obvious in usual dose of clinical use even if some lipidomics changes were happened.

Glycerophospholipids (GP) are among the most important lipid forming mammalian cell membranes. Deregulation of phospholipids can lead to many diseases [19-20]. Disruption of the phosphatidylcholine (PC) homeostasis is found in many neurodegenerative disorders [21]. These results suggest that the control of cellular phosphatidylcholine (PC) content, but not phosphotidylserine (PS) content, may be useful in the prevention or treatment of Alzheimer's disease [22]. Disrupted phospholipid homeostasis in ER(Endoplasmic reticulum) can lead to many neurological disorders, including schizophrenia and neurodegenerative diseases such as Alzheimer's, Parkinson's and Huntington diseases [23-24]. Sphingolipids, are a diverse class of lipids composed of free sphingoid bases and their phosphates, ceramides, and sphingomyelins, as well as complicated glycosphingolipids [25-26], which can be detected in miscellaneous diseases, including neurological diseases [27] and metabolic disorders [28]. The present data may provide a novel strategy for further exploration of the mechanism underlying inhalational anesthetics-evoked potential neurotoxicity in developing brain.

In our study, RNA sequencing (RNA-Seq) does not rely on a predesigned probe and enables rapid profiling and deep investigation of the transcriptome, for any tissues or species. GO(Gene Ontology) provides a systematic language to describe the attributes of genes, which are classified by cellular component, molecular function and biological process. This is the first report to evaluate RNA-seq changes in pregnant women for anesthetics exposure associated biomarkers. The qRT-PCR verification results indicated that Vcan was increased significantly in sevoflurane group, which indicate some transcriptomics changes was happened even if neural cell apoptosis was not significantly changed in sevoflurane group in comparison with control group in clinical use. Vcan were related with nervous system development [29]. Vcan may be capable of enhancing axonal and/or plasma membrane viability in culture, the complex microenvironment present in the stroked brain determines which genes are translated and trafficked accordingly [30]. Four different versions (isoforms) of the versican protein are produced from the Vcan gene. This versican protein likely helps regulate cell growth and division, the attachment of cells to one another (cell adhesion), and cell movement (migration). Studies suggest that versican plays a role in forming new blood vessels (angiogenesis) and inflammation. Versican also regulates the activity of several growth factors, which control a diverse range of processes important for cell growth. These isoforms (called V0, V1, V2, and V3) vary by size and by their location within the body. Versican interacts with many proteins and molecules to facilitate the assembly of the extracellular matrix and ensure its stability [30].

So UPLC/TOF-MS based lipidomics and RNA-seq provided comprehensive information for understanding pathological process of potential neurotoxicity caused by prenatal exposure to sevoflurane. Although further evidence is required, abnormal glycerophospholipid and sphingolipid metabolism may be used as an alternative way to understand the mechanism underlying inhalational anesthetics-induced neurotoxicity. Regulating glycerophospholipid and sphingolipid metabolism may be used as the potential therapeutic treatment for preventing inhalational anesthetics from potential impairing neurodevelopment. Meanwhile, Vcan gene change of sevoflurane group were significantly increased in the differential genes by qRT-PCR verification in offspring cerebral cortex.

\section{Conclusions}

In conclusion, the results of this study suggested lipid and RNA-seq changes associated with sevoflurane exposure during pregnancy and provides a better understanding of the risk in offspring exposed to sevoflurane even if there is no significant cell apoptosis changes in clinical dosage.

\section{Abbreviations}


HPLC, high performance liquid chromatography, UPLC, ultra performance liquid chromatography, TOF-MS, time-of-flight mass spectrometry, $\mathrm{PaO}{ }_{2}$, arterial partial pressure of oxygen, $\mathrm{PaCO}_{2}$, arterial partial pressure of carbon dioxide, RSDs, relative standard deviation, HEPA, high efficiency particulate air filter, ESI, electrospray ionization, MRM, multiple reaction monitoring, PLS-DA, partial least square-discriminant analysis, HE, hematoxylin and eosin staining, PBS, phosphate-buffered saline, FBS, fetal bovine serum, DAB, diaminobenzidine, BPC, base peak chromatogram, VIP, variable importance in the projection, PCA, principal component analysis, GPI, glycosylphosphatidylinositol, CNS, Central Nervous System, FNSCs, fetal neural stem cells, PKC, Protein Kinase C, GPL, glycerophospholipid, GP, glycerophospholipids, PC, phosphatidylcholine, PS, phosphotidylserine, RNA-seq, RNA sequencing, KEGG, Kyoto Encyclopedia of Genes and Genomes,

\section{Declarations}

\section{Ethics approval and consent to participate}

All animal studies, containing rats euthanasia procedures, were approved and complied with AALAC and IACUC guidelines and guidelines for institutional animal care in Shanghai Jiao Tong University (Permit number: A2016077).

\section{Acknowledgments}

We appreciated Professor Shikai Yan at the School of Pharmacy of Shanghai Jiao Tong University for providing us relevant comments and suggestions.

\section{Availability of data and materials}

All data supporting our findings are included in the manuscript.

\section{Funding}

This work was supported by the National Natural Science Foundation of China (No: 81401279), Shanghai Health and Family Planning Commission Foundation(No:20164y0263), Shanghai Natural Science Foundation(No: 18ZR1443100), Shanghai Jiao Tong Unviersity School of Medicine, Innovation center of Translational Medicine Collaboration(No: TM201729), Youth Talent Fund of International Peace Maternity and Child Health Hospital, Shanghai Jiaotong University School of Medicine in 2014 (To Zeyong Yang), Xinchen Foster Fund for Anesthesiologists in Shanghai (To Zeyong Yang) and China International Medical Exchange Fund(2019-anesthesia-14)

\section{Authors'contributions}

YZY and CZL initiated the project, JY, WY, HXX, MFH and LQ performed experiments, MQ and WYL participated in the experimental analyses, JY, CZL, and YZY discussed the interpretation of the relevant study, YZY and CZL supervised the study, JY, WY and YZY wrote the manuscript. All authors read and approved the final manuscript.

\section{Competing interests}

The authors declare that they have no competing interests.

\section{References}

1. Cattano D, Valleggi S, Abramo A, Forfori F, Maze M, Giunta F. Nitrous oxide discretely up-regulates nNOS and p53 in neonatal rat brain. Minerva Anestesiol. 2010,76: 420-4.

2. Akbar, M., Calderon, F., Wen, Z., Kim, H. Y. Docosahexaenoic acid: A positive modulator of Akt signaling in neuronal survival. Proc. Natl. Acad. SCl. U.S.A. 2005,102: 10858-63.

3. Huang, B. X., Akbar, M., Kevala, K., and Kim, H. Y. Phosphatidylserine is a critical modulator for Akt activation. J. Cell Biol. $2011,192: 979-92$.

4. Kim, H. Y., Akbar, M., Lau, A., and Edsall, L. Inhibition of neuronal apoptosis by docosahexaenoic acid (22:6n-3). Role of phosphatidylserine in antiapoptotic effect. J. Biol. Chem. 2000, 275: 35215-23.

5. Kim, H. Y. Novel metabolism of docosahexaenoic acid in neural cells. J. Biol. Chem. 2007,282: 18661-5.

6. Kim, H. Y., Akbar, M., and Kim, Y. S. Phosphatidylserinedependent neuroprotective signaling promoted by docosahexaenoic acid. Prostaglandins Leukot. Essent. Fatty Acids. 2010, 82: 165-72.

7. 7. Ma R, Wang X, Peng P, Xiong J, Dong H, Wang L, Ding Z. a-Lipoic acid inhibits sevoflurane-induced neuronal apoptosis through PI3K/Akt signalling pathway. Cell Biochem Funct. 2016,34:42-7.

8. Liu B, Gu Y, Xiao H, Lei X, Liang W, Zhang J. Altered metabolomic profiles may be associated with sevoflurane-induced neurotoxicity in neonatal rats. Neurochem Res. 2015,40:788-99.

9. van Meer G, de Kroon Al. Lipid map of the mammalian cell. J. Cell Sci. 2011, 124:5-8.

10. Rao Muralikrishna Adibhatla, J. F. Hatcher. Role of Lipids in Brain Injury and Diseases. Future Lipidol. 2007, 2: 403-22.

11. Yang Z, Lv J, Li X, Meng Q, Yang Q, Ma W, Li Y, Ke ZJ. Sevoflurane decreases self-renewal capacity and causes c-Jun N-terminal kinase-mediated damage of rat fetal neural stem cells. Sci Rep. 2017,7:46304. 
12. Zhao X, Yang Z, Joseph DJ, Inan S, Wei H. Dual Effects of Isoflurane on Proliferation, Differentiation, and Survival in Human Neuroprogenitor Cells. Anesthesiology. 2013, 118:537-49.

13. Zheng, H., Dong, Y., Xu, Z., Crosby, G., Culley, D., Zhang, Y., Xie, Z. Sevoflurane anesthesia in pregnant mice induces neurotoxicity in fetal and offspring mice. Anesthesiology. 2013,118: 516-26.

14. Suehara, T., Morishita, J., Ueki, M., Ueno, M., Maekawa, N., Mizobuchi, S. Effects of sevoflurane exposure during late pregnancy on brain development of offspring mice. Paediatr. Anaesth. 2016,26:52-9.

15. Fang, F., Song, R., Ling, X., Peng, M., Xue, Z., Cang, J. Multiple Sevoflurane Anesthesia in Pregnant Mice Inhibits Neurogenesis of Fetal Hippocampus via Repressing Transcription Factor Pax6. Life Sci. 2017,175:16-22.

16. Hong, S. et al. Soluble Abeta oligomers are rapidly sequestered from brain ISF in vivo and bind GM1 ganglioside on cellular membranes. Neuron. 2014,82:308-19.

17. Ruiz, A. Joshi P, Mastrangelo R, Francolini M, Verderio C, Matteoli M. Testing Abeta toxicity on primary CNS cultures using drug-screening microfluidic chips. Lab Chip. 2014,14:2860-6.

18. Shevchenko A, Simons K. Lipidomics: coming to grips with lipid diversity. Nat. Rev. Mol. Cell Biol. 2010,11: 593-8.

19. V.R. De, P.J.W.H. Kappelle, G.M. Dallinga-Thie, R.P.F. Dullaart, Plasma phospholipid transfer protein activity is independently determined by obesity and insulin resistance in non-diabetic subjects. Atherosclerosis. 2011,217: 253-9.

20. Igarashi M, Ma K, Gao F, Kim H, Rapoport SI, Rao JS. Disturbed choline plasmalogen and phospholipid fatty acid concentrations in Alzheimer's disease prefrontal cortex. J. Alzheimers Dis. 2011, 24: 507-17.

21. Morgan, N. V. et al. PLA2G6, encoding a phospholipase A(2), is mutated in neurodegenerative disorders with high brain iron. Nat. Genet. 2006, 38: 752-4.

22. Ko M, Hattori T, Abdullah M, Gong JS, Yamane T, Michikawa M. Phosphatidylcholine protects neurons from toxic effects of amyloid $\beta$-protein in culture. Brain Res. 2016, 1642: 376-83.

23. Adibhatla, R. M. \& Hatcher, J. F. Role of lipids in brain injury and diseases. Future lipidol. 2007, 2: 403-22.

24. Cheng, D., Jenner AM, Shui G, Cheong WF, Mitchell TW, Nealon JR., et al. Lipid pathway alterations in Parkinson's disease primary visual cortex. PLoS One. 2011,6: e17299.

25. Mullen TD, Hannun YA, Obeid LM. Ceramide synthases at the centre of sphingolipid metabolism and biology. Biochem. J. $2012,441: 789-802$.

26. Merrill Jr AH. Sphingolipid and glycosphingolipid metabolic pathways in the era of sphingolipidomics. Chem. Rev. 2011, 111: 6387-422.

27. Van Echten-Deckert G, Walter J. Sphingolipids: critical players in Alzheimer's disease. Prog. Lipid Res. 2012, 51: 378-93.

28. Hla T, Dannenberg AJ. Sphingolipid signaling in metabolic disorders. Cell Metab. 2012,16:420-34.

29. Wu, Y., Sheng, W., Chen, L., Dong, H., Lee, V., Lu F., et al. Versican V1 isoform induces neuronal differentiation and promotes neurite outgrowth. Mol. Biol. Cell 2004,15:2093-104.

30. Chiarelli N, Carini G, Zoppi N, Ritelli M, Colombi M. Transcriptome analysis of skin fibroblasts with dominant negative COL3A1 mutations provides molecular insights into the etiopathology of vascular Ehlers-Danlos syndrome. PLoS One. 2018,13:e0191220.

31. Kanehisa M, Furumichi M, Mao T, Sato Y, Morishima K. KEGG: new perspectives on genomes, pathways, diseases and drugs. Nucleic Acids Res.2017,45:D353-61.

32. Kanehisa M, Sato Y, Kawashima M, Furumichi M, Mao T. KEGG as a reference resource for gene and protein annotation. Nucleic Acids Res. 2016,44:D45762.

33. Kanehisa M, Goto S. KEGG: Kyoto encyclopedia of genes and genomes. Nucleic Acids Res. 2000,28:27-30.

34. Mao X, Cai T, Olyarchuk JG, Wei L. Automated genome annotation and pathway identification using the KEGG orthology (KO) as a controlled vocabulary. Bioinformatics. 2005,21:3787-93.

35. Storey JD. The positive false discovery rate: a bayesian interpretation and the q-value. Ann Stat. 2003,31:2013-35.

\section{Tables}

Table 1. Arterial blood gas analysis for two groups.

\begin{tabular}{|lll|}
\hline & C group $(n=8)$ & S group $(n=8)$ \\
\hline $\mathrm{PH}$ & $7.37 \pm 0.03$ & $7.41 \pm 0.01$ \\
\hline $\mathrm{PaCO}_{2}(\mathrm{mmHg})$ & $43.80 \pm 3.50$ & $46.97 \pm 4.25$ \\
$\mathrm{PaO}_{2}(\mathrm{mmHg})$ & $116.0 \pm 12.66$ & $124.3 \pm 19.59$ \\
$\mathrm{BE}$ & $-0.33 \pm 1.53$ & $4 \pm 1.0$ \\
$\mathrm{HCO}_{3}$ & $25.57 \pm 1.75$ & $29.90 \pm 1.90$ \\
$\mathrm{SaO}_{2}(\%)$ & $98.33 \pm 0.58$ & $100 \pm 0$ \\
\hline
\end{tabular}

Table 1. Arterial blood gas analysis for two groups. There are no significance for two groups. $\mathrm{PH}$ value, partial pressure of oxygen $\left(\mathrm{PaO}_{2}\right)$, partial pressure of carbon dioxide $\left(\mathrm{PaCO}_{2}\right), \mathrm{HCO}_{3}, \mathrm{BE}$ and $\mathrm{SaO}_{2}$ are monitored. 
Table 2 Potential bimarkers and related pathways or diseases.

\begin{tabular}{|c|c|c|c|c|c|c|c|}
\hline No. & & $M / Z$ & $\mathrm{RT}(\min )$ & Ion & Metabolite & Related Pathways & $\begin{array}{l}\text { Related } \\
\text { Disease }\end{array}$ \\
\hline 1 & 1687 & 496.341 & 2.27 & {$[\mathrm{M}+\mathrm{H}]+$} & LysoPC(16:0) & $\begin{array}{l}\text { glycerophospholipid } \\
\text { metabolism } \star \star \star\end{array}$ & $\begin{array}{l}\text { pancrea } \\
\text { cancer冈I } \\
\text { sgl囚 } \\
\text { atherosı }\end{array}$ \\
\hline 2 & 2918 & 707.4991 & 2.96 & {$[\mathrm{M}+\mathrm{K}]+$} & DG(20:1n9/0:0/20:5n3) & & \\
\hline 3 & 3488 & 774.5638 & 7.86 & {$[\mathrm{M}+\mathrm{H}]+$} & PE(22:6(4Z,7Z,10Z,13Z,16Z,19Z)/P-18:1(11Z)) & $\begin{array}{l}\text { glycerophospholipid } \\
\text { metabolism }{ }^{\star \star}\end{array}$ & $\begin{array}{l}\text { pancrea } \\
\text { cancer囚l } \\
\text { sgl }\end{array}$ \\
\hline 4 & 3037 & 728.5217 & 8.57 & {$[\mathrm{M}+\mathrm{Na}]+$} & $\operatorname{SM}(\mathrm{d} 18: 0 / 16: 0)$ & $\begin{array}{l}\text { sphingolipid } \\
\text { metabolism** }\end{array}$ & atherose \\
\hline 5 & 3929 & 808.5897 & 8.85 & {$[\mathrm{M}+\mathrm{H}]+$} & $\mathrm{PC}(16: 0 / 22: 5(7 Z, 10 Z, 13 Z, 16 Z, 19 Z))$ & $\begin{array}{l}\text { glycerophospholipid } \\
\text { metabolism }^{\star *}\end{array}$ & \\
\hline 6 & 3897 & 806.5729 & 9.08 & {$[\mathrm{M}+\mathrm{Na}]+$} & Galactosylceramide (d18:1/22:0) & $\begin{array}{l}\text { sphingolipid } \\
\text { metabolism** }\end{array}$ & $\begin{array}{l}\text { Hidrade } \\
\text { suppura }\end{array}$ \\
\hline 7 & 3535 & 780.5505 & 9.13 & {$[\mathrm{M}+\mathrm{Na}]+$} & CerP(d18:1/26:0) & & \\
\hline 8 & 3238 & 756.5559 & 9.8 & {$[\mathrm{M}+\mathrm{Na}]+$} & $\mathrm{PC}(16: 0 / 16: 0)$ & $\begin{array}{l}\text { glycerophospholipid } \\
\text { metabolism } \star \star\end{array}$ & $\begin{array}{l}\text { Adolesc } \\
\text { idiopath } \\
\text { scoliosi: }\end{array}$ \\
\hline 9 & 5586 & 923.7476 & 22.92 & {$[\mathrm{M}+\mathrm{H}]+$} & TG(20:5(5Z,8Z,11Z,14Z,17Z)/18:2(9Z,12Z)/20:5(5Z,8Z,11Z,14Z,17Z)) & & \\
\hline 10 & 4776 & 857.7568 & 23.98 & {$[\mathrm{M}+\mathrm{K}]+$} & TG(14:0/20:1(11Z)/15:0) & & \\
\hline
\end{tabular}

Table 2. RT: retention time(minute). M/Z: mass-to-charge ratio. VIP: variable importance in the projection. Student's $t$-test was performed by Graph Prism 5.0 , $p$-values were considered statistically significant if they were $*<0.05, * * P<0.01$.

\section{Figures}

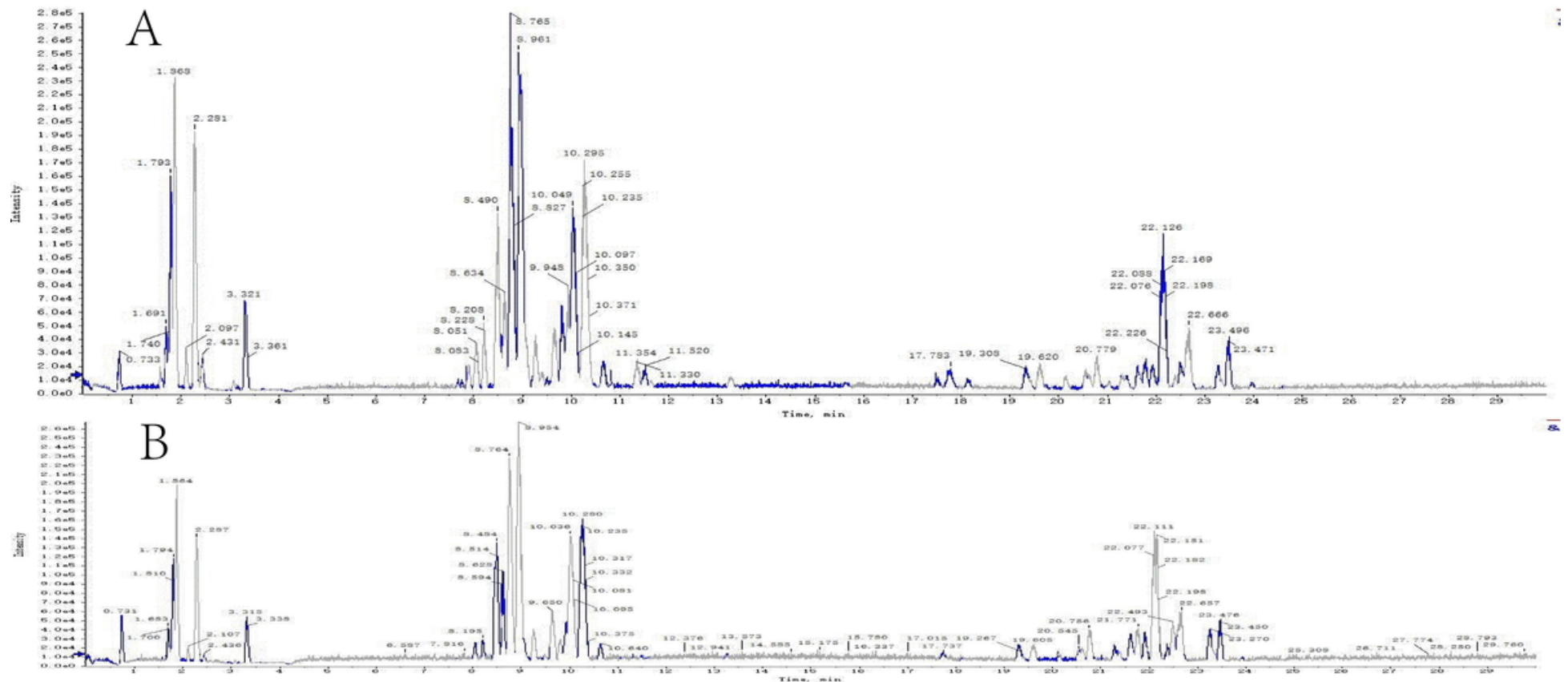

\section{Figure 1}

Typical base peak intensity (BPI) chromatogram of the rat serum obtained in ESI positive and negative mode based on UPLC/TOF-MS analysis. (a, b) Representative BPI chromatogram of rat serum in ESI positive and negative mode, respectively. The UPLC/TOF-MS analysis was performed using an Acquity TMUPLC system (Waters Corporation) coupled to a SynaptTMG2 High-Definition Time-of-Flight Mass Spectrometry system (WatersCorporation) with electrospray ionization (ESI) in positive and negative modes. In both positive and negative ion modes, BPC profiles display no difference between S and C groups. 
A
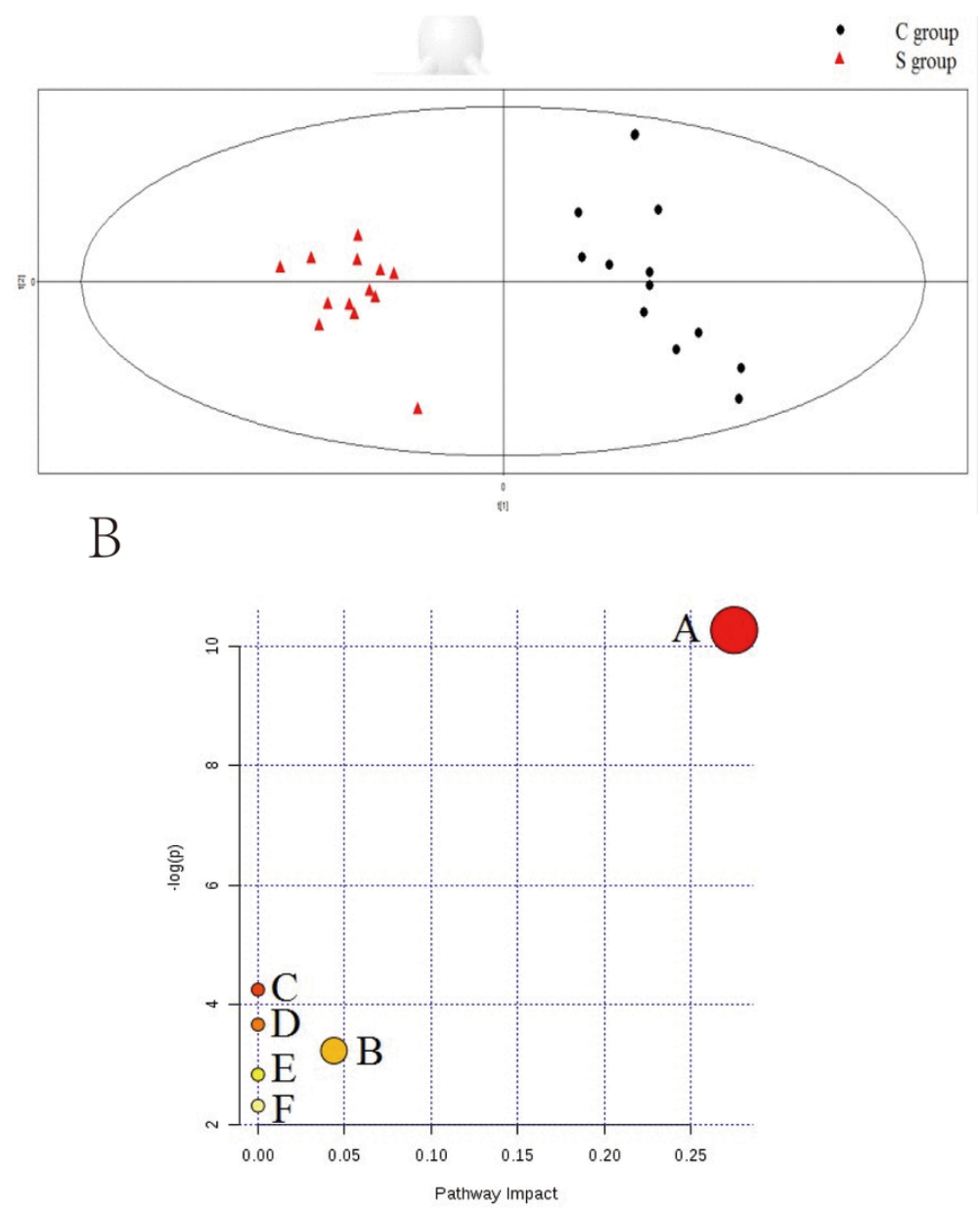

\section{Figure 2}

2a. C: PLS-DA in negative ion mode. S: PLS-DA in positive ion mode. In Cand S panel, the black boxes (.) represent samples from C group, the open triangles $(\triangle)$ represent samples from S group. $2 b$. Pathway analysis on biomarkers of sevoflurane-induced neurogenerative disease model. All matched pathways were acquired according to $p$-values from pathway enrichment analysis and pathway impact values from pathway topology analysis, using pathway library of Rattus norvegicus (rat). A. Glycerophospholipid metabolism, B.Glycosylphos phatidylinositol (GPI)-anchor biosynthesis Linoleic acid metabolism, C. AlphaLinolenic acid metabolism, D. Sphingolipid metabolism, E. Arachidonic acid metabolism.

A

A

Tunel

Blank Ctrl Hippocampus

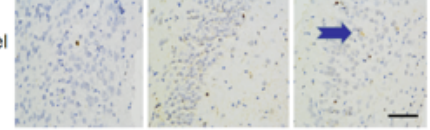

HE

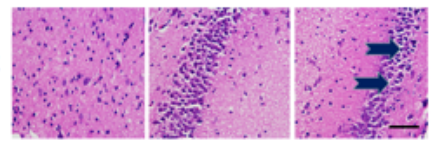

B

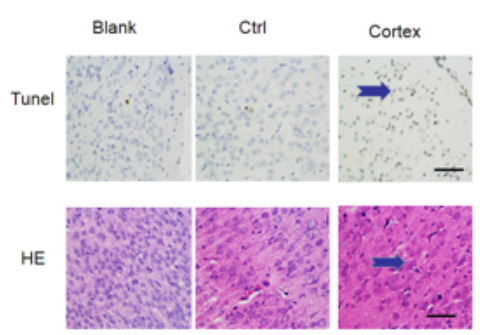

C

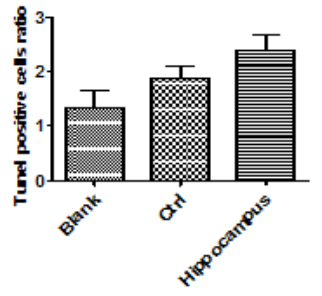

D

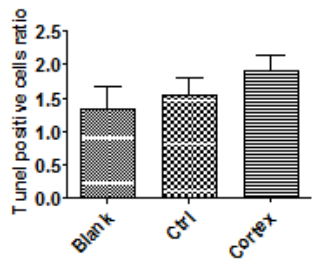

Figure 3 
Observation of HE and TUNEL staining for the hippocampus and cerebral cortex tissue in morphological changes in each group $(\times 400)(3 a, 3 b) .$. Scale bar $=$ $200 \mu \mathrm{m}$. The statistical analysis of neural cell apoptosis was detected by TUNEL and HE staining from hippocampi and cerebral cortex tissue of each offspring rat (3c, 3d). All data were derived from Tunel staining results of independent experiments. Values are the mean \pm S.D. of triplicate experiments, and analyzed by ANOVA with Tukey post hoc tests. *P囚0.05 compared to control group.
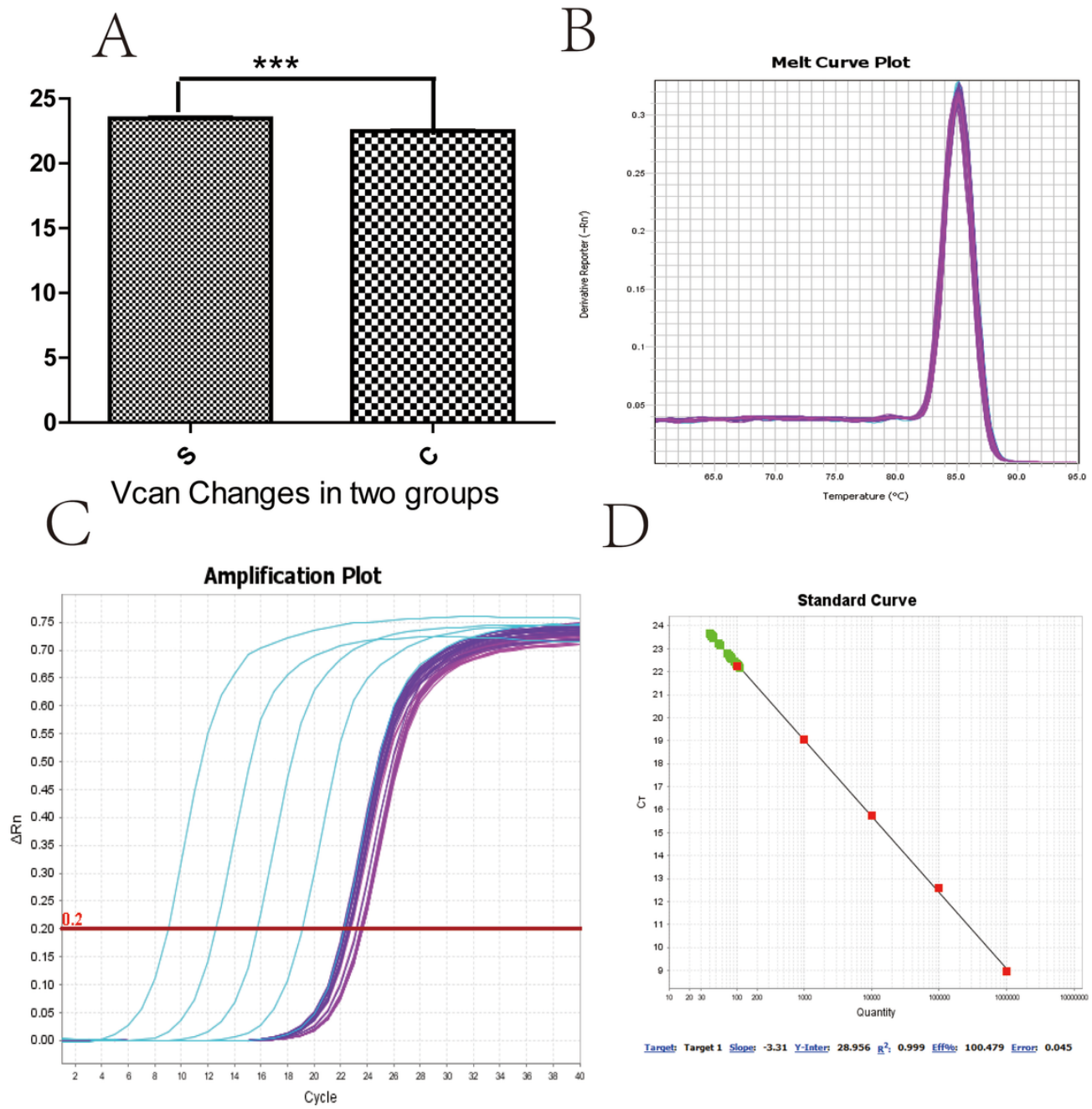

Figure 4

Vcan gene RT-PCR changes, included melting, amplification and standard curves. 4a for RT-PCR changes of Vcan gene, 4b for Melt Curve Plot Vcan, 4c for Amplification Plot Vcan, and 4d for Standard Curve Vcan. Values are the mean \pm S.D. of triplicate experiments, and analyzed by ANOVA with Tukey post hoc tests. *Pख0.05 compared to control group. 

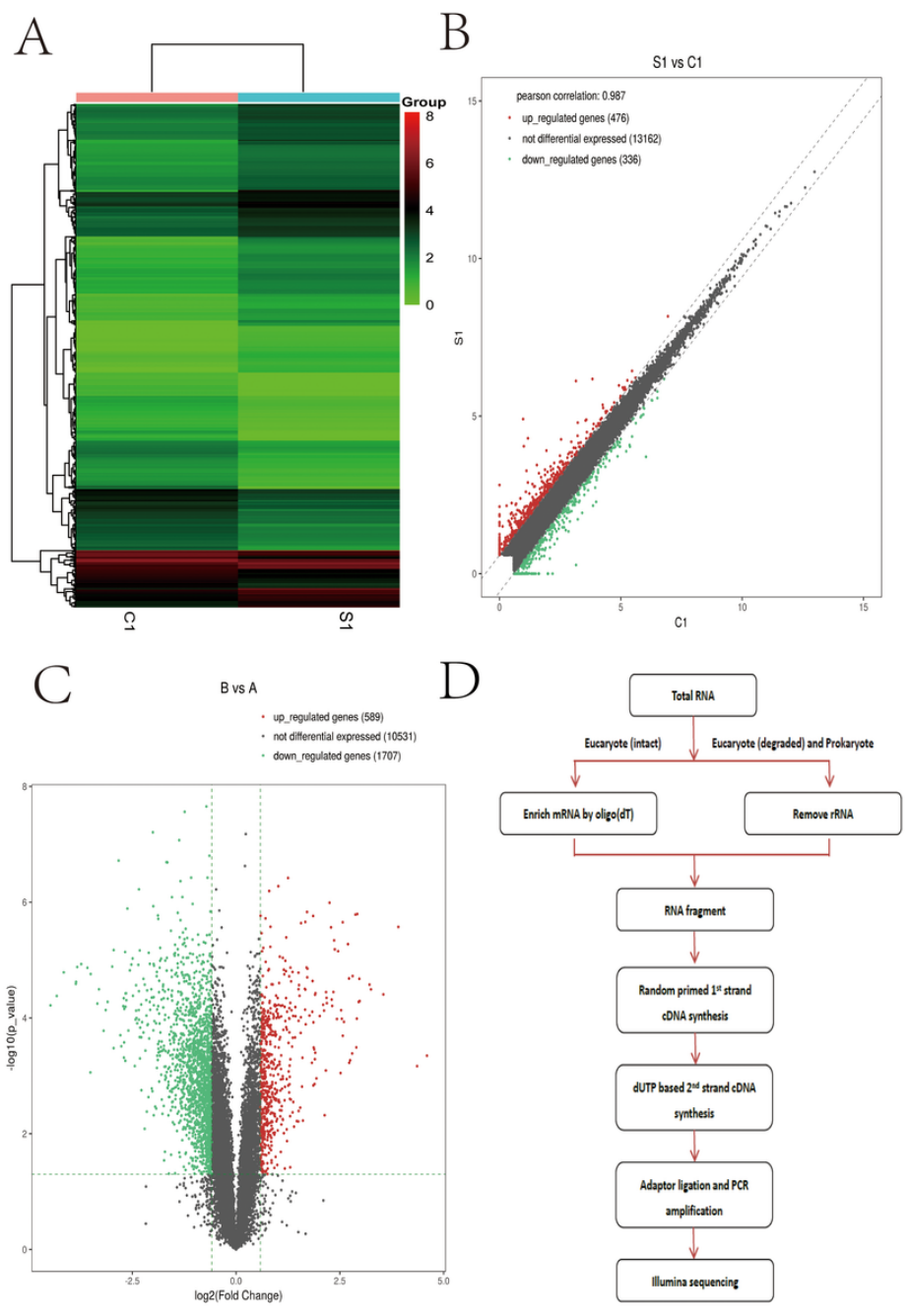

\section{Figure 5}

5a, Significantly differentially expressed gene cluster thermogram. Differential expression gene cluster map. Each row represents a gene and each column represents a sample. Red represents upregulation of significantly differentially expressed genes, while green represents down-regulation of significantly differentially expressed genes. 5b, mRNA scatter diagram. The $x$ axis and $y$ axis indicated the average FPKM value (log2 transformation) of the samples, the red dots upregulated the differentially expressed genes, the green dots down-regulated the differentially expressed genes, and the gray dots represented no differentially expressed genes. Two oblique dotted lines were divided into upper, down-regulated genes ( 1.5 times difference) and unaltered genes. $5 \mathrm{c}$, mRNA Volcanic map, The $x$ axis represents the log2Fold_Change value, and the $y$ axis denotes-log10p_value. The vertical two green lines were up-regulated (right) and down-regulated (left), respectively. The green parallel line corresponded to p-value threshold. Green dots represent differentially down-regulated genes, red dots represent differentially up-regulated genes, and gray spots represent non-significant differentially differentiated genes. $5 \mathrm{~d}$. RNA-seq experimental flow chart. 


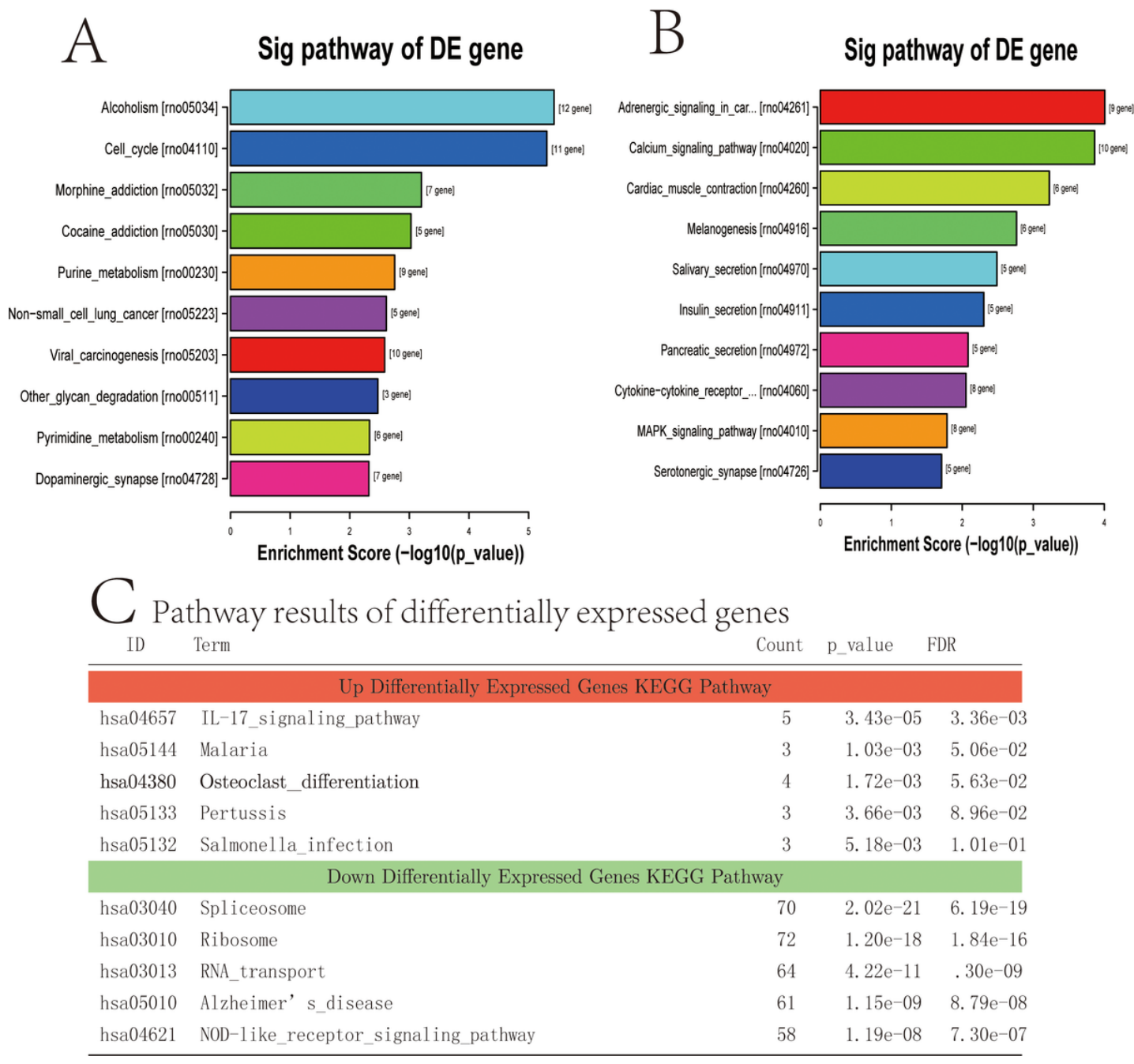

\section{Figure 6}

Enrichment analysis of KEGG Pathway. a. b. The KEGG pathway analyzed the top 10 item bar diagrams. In p-value order from low to high, the ordinate represents P-value (- log10 transformation) c. Pathway Analysis of the first 5 Pathway of up and down significantly differentially expressed genes KEGG Pathway. Pathway analysis is a functional analysis mapping genes to KEGG pathways. The p-value (EASE-score, Fisher-P value or Hypergeometric-P value) denotes the significance of the Pathway correlated to the conditions. Lower the p-value, more significant is the Pathway. (The recommend p-value cut-off is 0.05.) Upregulated differential expression Gene KEGG Pathway Analysis results folder: Up, down-regulated differential expression Gene KEGG Pathway Analysis results folder: Down.

\section{Supplementary Files}

This is a list of supplementary files associated with this preprint. Click to download.

- SupplementalTable1bloodgas.docx

- SupplementalTable2Potentialbiomarkers.docx 